\title{
Experimentelles Studium der inneren Sekretion des Pankreashormons.
}

\author{
IX. Über die Ausscheidung von Pankreashormon und \\ einige Einflüsse desselben auf die Galle. \\ Von \\ Kishiro Kozuka. \\ (小塚喜四郎) \\ (Aus Prof. Kumagai's medizinischer Klinik an der \\ Tohoku Universität zu Sendai.)
}

In früheren Arbeiten ${ }^{129}$ haben wir berichtet, dass das künstlich extrahierte Pankreashormon durch verschiedene Stellen vom tierischen Körper resorbiert wird und eine blutzuckersenkende Wirkung entfaltet, obwohl die Wirkungsdauer und Intensität desselben je nach den Umständen verschieden sind. Wie verhält sich nun das resorbierte Pankreashormon bei Überschuss im Körper? I $\mathrm{ch}^{3)}$ hatte bereits mitgeteilt, dass man die blutzuckersenkende Substanz aus den Harnen der meisten normalen Menschen, aber nicht aus dem Diabetikerharn isolieren kann, während sie in dem mit Pankreashormon zuckerfrei gemachten Diabetikerharn nachweisbar ist, und ich hatte daraus den Schluss gezogen, dass die Hypoglykämie erzeugende Substanz in menschlichen Harn sehr wahrscheinlich mit Pankreashormon identisch ist und der überschüssige Anteil des Pankreashormons für den Kohlenhydratstoffwechsel von der Niere ausgeschieden wird. Das Pankreashormon wird also in den Harn ausgeschieden. Wird es aber daneben nicht in die Galle ausgeschieden, wie es bei verschiedenen einverleibten Arzneimitteln ader Stoffwechselprodukten in Körper geschieht? Wenn diese Annahme richtig wäre, so müsste dementsprechende Zunahme der die Hypoglykïmie erzeugenden Substanz in der Galle nach der Hormoninjektion nachgewiesen werden. Um diese Frage zu entscheiden, ist es nötig zuerst zu erkennen, ob solcher Stoff in der normalen Galle enthalten ist oder nicht.

\footnotetext{
1) Katsura und Kozuka, Tohoku Jour. of Exper. Med., 1929, 12, 241.

2) Kozuka, Tohoku Jour. of Exper. Med., 1929, 12, 354.

3) Kozuk a, Tohoku Jour. of Exper. Med., 1927, 9, 130.
} 


\section{Isolierung der blutzuckersenkenden Substanz aus der Galle.}

Verschiedene Methoden sind von mehreren Autoren erdacht, um die blutzuckersenkende Substanz aus den tierischen Geweben oder Organen sowie aus den pflanzlichen Materialien zu gewinnen, aber es gibt keine einzige unter ihnen, welche erfolgreich bei allen Füllen gültig ist. Es ist vielfach von den Medien abhängig, aus welchem es isoliert wird. Darum hatte ich im voraus geprüft, welche Methode für die Galle am zweckmässigsten ist. Wir hatten das rohe Pankreasextrakt in bestimmter Menge zur frischen Schweinegalle getan und mit verschiedenen Methoden untersucht, wie viel des zugesetzten Pankreashormons aus diesem Gemische sich zurückgewinnen lässt. Über Einzelheiten der hier angewandten Methoden findet man in meiner früheren Arbeit. ${ }^{3)}$

1. Schwefelammonmethode: 4 Kanincheneinheiten wurden nit 80 ccm Galle gemischt. Das Extrakt zeigt mittels dieser Methode bei Kaninchen 4 Stunden nach der Injektion 16\% ige Erniedrigung des Blutzuckers.

2. Gerbsäuremethode: Die gleiche Menge Pankreashormon wie beim vorigen Fall ist in die Galle getan, deren Extrakt um $21 \%$ ige Hypoglykämie bewirkt.

3. Pikrinsäuremethode: Das durch diese Methode gewonnene Extrakt aus der Galle, die 4 Kanincheneinheiten entbält, vermag den Blutzucker um $28 \%$ zu senken.

4. Karbal-Schwefelsäuremethode: Bei Zusatz von 2 Kanincheneinheiten in die Galle erweist das Extrakt 26\%óige Herabsetzung des Blutzuckers.

5. Schwefelsäure-Alkoholmethode: Das nach dieser Methode isolierte Extrakt aus der Galle, zu welcher 2 Kanincheneinheiten eingetragen sind, zeigt $57 \%$ ige Hypoglylämie, d. h. die Wirkung von etra einer Kanincheneinheit.

Die wichtigen Punkte dieser Versuchsreihe sind in der Tabelle I zusammengefasst und man kamn daraus ersehen, dass die Alkoholmethote bezüglich der Galle die andern 4 Mcthoden übertrifft, und dass sich durch diese Methode wenigstens die halbe Menge vom zugesetzten Pankreashormon zurückgewinnen lässt. Freilich muss ich hier betonen, dass auch in der normalen Galle kleine Menge von blutzuckersenkender Substanz enthalten ist, worüber ich im nächsten Kapital noch einmal sprechen werde. 


\section{Tabelle I.}

Vergleichung verschiedener Methoden.

\begin{tabular}{|c|c|c|c|c|c|c|c|c|c|c|}
\hline \multirow[b]{2}{*}{ Nr. } & \multirow[b]{2}{*}{ Methode } & \multirow{2}{*}{ 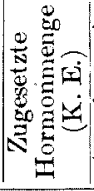 } & \multirow{2}{*}{ 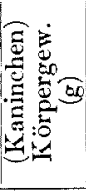 } & \multicolumn{6}{|c|}{ Blutzucker $(\%)$} & \multirow[b]{2}{*}{ 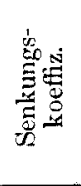 } \\
\hline & & & & $\begin{array}{l}\dot{\vec{c}} \cdot \dot{\vec{\Xi}} \\
\ddot{\vec{p}}\end{array}$ & 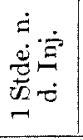 & 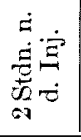 & 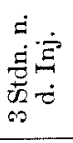 & 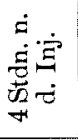 & 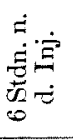 & \\
\hline 1 & $\begin{array}{l}\text { Schwefel- } \\
\text { ammonium }\end{array}$ & 4,0 & 1820 & 0,107 & 0,117 & 0,106 & 0,104 & 0,098 & 0,090 & $-16 \%$ \\
\hline 2 & Gerbsiiure & 4,0 & 2000 & 0,092 & 0,090 & 0,089 & 0,073 & 0,074 & 0,085 & $-21 \%$ \\
\hline 3 & Pikrinsiure & 4,0 & 1830 & 0,085 & 0,067 & 0,061 & 0,063 & 0,070 & 0,075 & $-23 \%$ \\
\hline 4 & $\begin{array}{l}\text { Karbol-Schwe- } \\
\text { felsiure }\end{array}$ & 2,0 & 1720 & 0,108 & 0,084 & 0,080 & 0,082 & 0,086 & $0,0 \mathrm{~s} s$ & $-26 \%$ \\
\hline 5 & $\begin{array}{c}\text { Schwefelsïure- } \\
\text { Alkohol }\end{array}$ & 2,0 & 1900 & 0,094 & 0,088 & 0,079 & 0,050 & 0,040 & & $-57 \%$ \\
\hline
\end{tabular}

Die Hypoglykämie erzeugende Substanz in der normalen Schweinegalle.

Aus der frischen Blasengalle von Schwein wird das Extrakt mittels der Alkoholmethode dargestellt und einem vorher gefasteten Kaninchen subkutan gespritzt. Die Gallenmenge der einzelnen Blase schwankt ziemlich bedeutend, nämlich zwischen 57 und $120 \mathrm{ccm}$. Die Resultate sind in der Tabelle II angegeben. Bei Fall 3 und 8 sieht man keine bemerkbare Veränderung des Prozentgehalts von Blutzucker, während bei den anderen 6 Fällen auffallende Senkung vorkommt. Es ist aus dieser Tabelle verständlich, dass die blutzuckersenkende Substanz in den meisten normalen Gallen enthalten ist. Dass in einigen Fällen keine Hypoglykümie erzeugende Substanz nachgewiesen wird, bedeutet nicht, dass solche Substanz in der Galle fehlt, weil sie durch diese Methode nur in halber Menge herausgenommen wird.

Hypoglykämie erzeugende Substanz in Harn und Galle von normalen Kaninchen.

Ferner hatte ich geprüft, ob die Hypoglykämie erzeugende Substanz in Harn sowie in Galle des normalen Kaninchens vorhanden ist oder nicht. 
Der Harn wird durch Katheterisation gesammelt und nach der Gerbsäuremethode extrahiert. Die Galle wurde in folgender Weise gewonnen ; man hat das Kaninchen auf dem Operationstische gefesselt, die Bauchhöhle in der Mittellinie oberhalb des Nabels aufgemacht, den Ductus choledochus in der Nähe der Einmündungsstelle in Duodenum unterbunden und dann eine Kanüle, welche mittels eines Gummirohrs mit in der Mitte ausgebauchtem und leicht gebogenem Glasrohr kommuniziert, in denselbe eingelegt. Dann wird die Öffnung geschlossen, indem man die Künüle an der Haut und Muskelschicht zusammennüht, um vor Abrutschen zu hüten. Die für die Operation nötige Zeit beträgt ca. 10-20 Minuten. Die ausgeschiedene Galle sammelt sich durch so eingerichtete Kanüle in der Ampulle.

Fall 1. Kaninchen, $2150 \mathrm{~g}$. In 5 Stunden gewann ich $27,9 \mathrm{ccm}$ Galle, deren Extrakt 13\% ige Erniedrigung des Blutzuckers bervorrief.

Fall 2. Kaninchen, $2000 \mathrm{~g}$. Während 2 Stunden entleerte das Kaninchen $14,0 \mathrm{ccm}$ Galle und $30,0 \mathrm{ccm}$ Harn. Das Extrakt aus diesen zwei Stoffen erzeugte 17\% ópige resp. 14\% \%ige Hypoglykämie.

Fall 3. Kaninchen, $2000 \mathrm{~g}$. Wiihrend 9 Stunden wurden $80,0 \mathrm{~cm}$ Galle und $37,0 \mathrm{ccm}$ Harn ausgeschieden. Bei jedem Extrakt konmt mässige Hypoglykïmie zustande, ninnlich $16 \%$ beim ersteren und $13^{\circ} \%$ beim letzteren.

Wir kommen aus diesen Tersuchen, die in der Tabelle II genan zusammengestellt sind, zum Schluss, das das Extrakt sowohl aus der Galle als auch aus dem Harn rom normalen Kaninchen hypoglyiämisch einwirken kann.

\section{Tabelle II.}

Die Hypogrlykäinie erzeugende Substanz in der normalen Schweine- und Kaninchengalle und Kaninchenharn.

\begin{tabular}{|c|c|c|c|c|c|c|c|c|c|c|c|}
\hline \multirow{2}{*}{$\perp$} & & \multirow{2}{*}{\multicolumn{2}{|c|}{$\begin{array}{c}\text { Material u. } \\
\text { Menge } \\
\text { (ecni) }\end{array}$}} & \multirow[b]{2}{*}{ 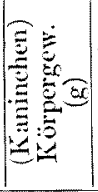 } & \multicolumn{6}{|c|}{ Blutzucker $(0.0)$} & \multirow[b]{2}{*}{ 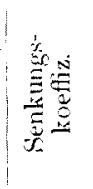 } \\
\hline & & & & & $\dot{\vec{\Xi}}$ & 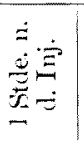 & 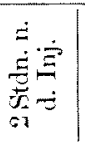 & $\begin{array}{l}\dot{\Xi} \\
\dot{\vec{\Xi}} \\
\dot{\vec{\Xi}} \\
\dot{D}\end{array}$ & 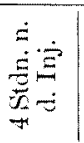 & $\begin{array}{l}\dot{\vec{E}} \\
\dot{\vec{E}} \\
\dot{\vec{S}} \\
\dot{0}\end{array}$ & \\
\hline$\frac{\Xi}{\frac{E}{E}}$ & $\begin{array}{l}2 \\
3 \\
4 \\
5 \\
6 \\
7\end{array}$ & $\begin{array}{c}\text { Gialle } \\
\text { " } \\
" \\
" \\
" \\
" \\
"\end{array}$ & $\begin{array}{r}82,0 \\
57,0 \\
95,0 \\
80,0 \\
78,0 \\
120,0 \\
87,0 \\
84,0\end{array}$ & $\begin{array}{l}1740 \\
1760 \\
1800 \\
1300 \\
1880 \\
1800 \\
1600 \\
1550\end{array}$ & $\begin{array}{l}0,115 \\
0,098 \\
0,089 \\
0,120 \\
0,107 \\
0,110 \\
0,096 \\
0,081\end{array}$ & $\begin{array}{l}0,104 \\
0,114 \\
0,087 \\
0,118 \\
0,098 \\
0,106 \\
0,087 \\
0,083\end{array}$ & $\begin{array}{l}0,097 \\
0,109 \\
0,087 \\
0,098 \\
0,089 \\
0,102 \\
0,089 \\
0,087\end{array}$ & $\begin{array}{l}0,098 \\
0,098 \\
0,094 \\
0,105 \\
0,092 \\
0,100 \\
0,098 \\
0,076\end{array}$ & $\begin{array}{l}0,090 \\
0,094 \\
0,089 \\
0,107 \\
0,098 \\
0,098 \\
0,099 \\
0,079\end{array}$ & $\begin{array}{l}0,090 \\
0,087 \\
0,111 \\
0,100 \\
0,094 \\
0,079\end{array}$ & $\begin{array}{r}-20 \% \\
-120 \\
1- \\
-190 \\
-170 \\
-150 \\
-10 \% \\
1\end{array}$ \\
\hline 五 & 3 & $\begin{array}{l}\text { Galle } \\
\text { fGalle } \\
\text { IFarn } \\
\text { jGalle } \\
\text { iIIarn }\end{array}$ & $\begin{array}{l}27,9 \\
14,0 \\
30,0 \\
80,0 \\
37,0\end{array}$ & $\begin{array}{l}2000 \\
1850 \\
1920 \\
1950 \\
2050\end{array}$ & $\begin{array}{l}0,104 \\
0,106 \\
0,099 \\
0,101 \\
0,100\end{array}$ & $\begin{array}{l}0,095 \\
0,102 \\
0,097 \\
0,102 \\
0,100\end{array}$ & $\begin{array}{l}0,091 \\
0,097 \\
0,086 \\
0,090 \\
0,092\end{array}$ & $\begin{array}{l}0,090 \\
0,058 \\
0,057 \\
0,085 \\
0,096\end{array}$ & $\begin{array}{l}0,099 \\
0,096 \\
0,109 \\
0,099 \\
0,093\end{array}$ & $\begin{array}{l}0,102 \\
0,091\end{array}$ & $\begin{array}{l}-1800 \\
-1700 \\
-1400 \\
-100 \\
-130\end{array}$ \\
\hline
\end{tabular}




\section{Quantitative Veränderung der blutzuckersenken- den Substanz in Galle und Harn nach der \\ Injektion von Pankreashormon.}

Ich konnte die den Blutzucker senkende Substanz in Galle und Harn vom normalen Kaninchen nachweisen, obwôhl in kleiner Menge. Wenn nun das Pankreashormon bei Überschuss für Assimilation von Kohlenhydraten in die Galle ausser dem Harn abgesondert würde, so müsste man stärker wirksames Extrakt aus der Galle nach der Hormoninjektion herzustellen vermögen als vor derselben, weil der präexsistierende Anteil mit dem ausgeschiedenen zusammen herausgenommen wird. Ich hatte den Harn und die Galle je vor und nach der Hormoninjektion angesammelt und jedes Extrakt auf die blutzuckersenkende Wirkung geprüft.

Fall 1. Kaninchen, $2000 \mathrm{~g}$. Ehe die Operation ausgeführt wurde, wurde $19,0 \mathrm{ccm}$ Harn in normalem Zustand katheterisiert und zur Extraktion benutzt. Ich hatte $4 \mathrm{Ka}$ nincheneinheiten Pankreashormon 3mal eingeteilt subkutan gespritzt und danach 47,1 ccu Galle im Laufe von 6 Stunden und 20 Minuten bekommen, deren Extrakt 9\%ige Verminderung des Blutzuckers zeigt; man kann nämlich den Einfluss von Hormoninjektion auf den Gehalt an hypoglylä̈mischer Substanz in der Galle nicht konstatieren. Dagegen erweist das Extrakt aus dem Harn nach der Injektion 13\%ige Hypoglylïmie, wïhrend bei dem vor derselben keine Veränderung des Zuekergehaltes zustande kommt.

Fall 2. Kaninchen, $2400 \mathrm{~g}$. Nach dem Ansammeln von $8,0 \mathrm{ccm}$ Harn und $13,2 \mathrm{ccm}$ Galle in 80 Minuten wurden $7 \mathrm{Kanincheneinheiten} 4 \mathrm{mal}$ verteilt subkutan appliziert. Nach der Injektion konnten wir $77,5 \mathrm{ccm}$ Galle und $11,0 \mathrm{ccm}$ Harn in 7 Stunden gewinnen. Trotzdem das Extrakt aus beiden vor der Einverleibung keinesfalls Hypoglykümie verursacht, zeigt dasjenige aus der Galle nach der Injektion vielmehr Hyperglykämie, während auf die Injektion desjenigen aus dem Harn nach der Pankreashormoninjektion 1s\%ige Erniedrigung exfolgt.

Fall 3. Kaninchen, $1790 \mathrm{~g}$. 47,0 ccm Galle und 53,0 ccm Harn werden im Laufe von 9 Stunden ausgeschieden. Danach bekommt das Kaninchen $2 \mathrm{mal}$ je 2 Kanincheneinheiten subkutan. Nach der Injektion konnte ich nur $8,3 \mathrm{ccm}$ Galle, aber leider keinen Harn entnehmen. In der Tabelle III ist die Wirkung jedes Extraktes angegeben. Wenn man dieselbe aus der Galle vor und nach der Injektion vergleicht, so kann man keinen bemerkbaren Unterschied wahrnehmen.

Fall 4. Kaninchen, $1980 \mathrm{~g}$. Gallenmenge vor und nach der Injektion beträgt je 57,4 $\mathrm{ccm}$ in 5 Stunden und $21,0 \mathrm{~cm}$ in 3 Stunden. Jedes Extrakt erzeugt je $8 \%$ ige und $12 \%$ ige Herabsetzung von Blutzuckergehalt. Betreffs des Harns bemerkt man, dass das Extrakt vor der Injektion hyperglykïmisch wirkt, wïhrend das nach derselben 22\%ige Senkung aufweist.

Wenn man die oben genannten Resultate betrachtet, so wird man erkennen, dass in der normalen Galle eine Hypoglykämie erzeugende Substanz, wenn auch in geringer Menge, enthalten ist, die aber durch Hormoninjektion keine Vermehrung erfährt, und dass man diejenige Substanz aus dem normalen Harn von Kaninchen extrahieren kann, die im Gegensatz 
Innere Sekretion des Pankreas. IX.

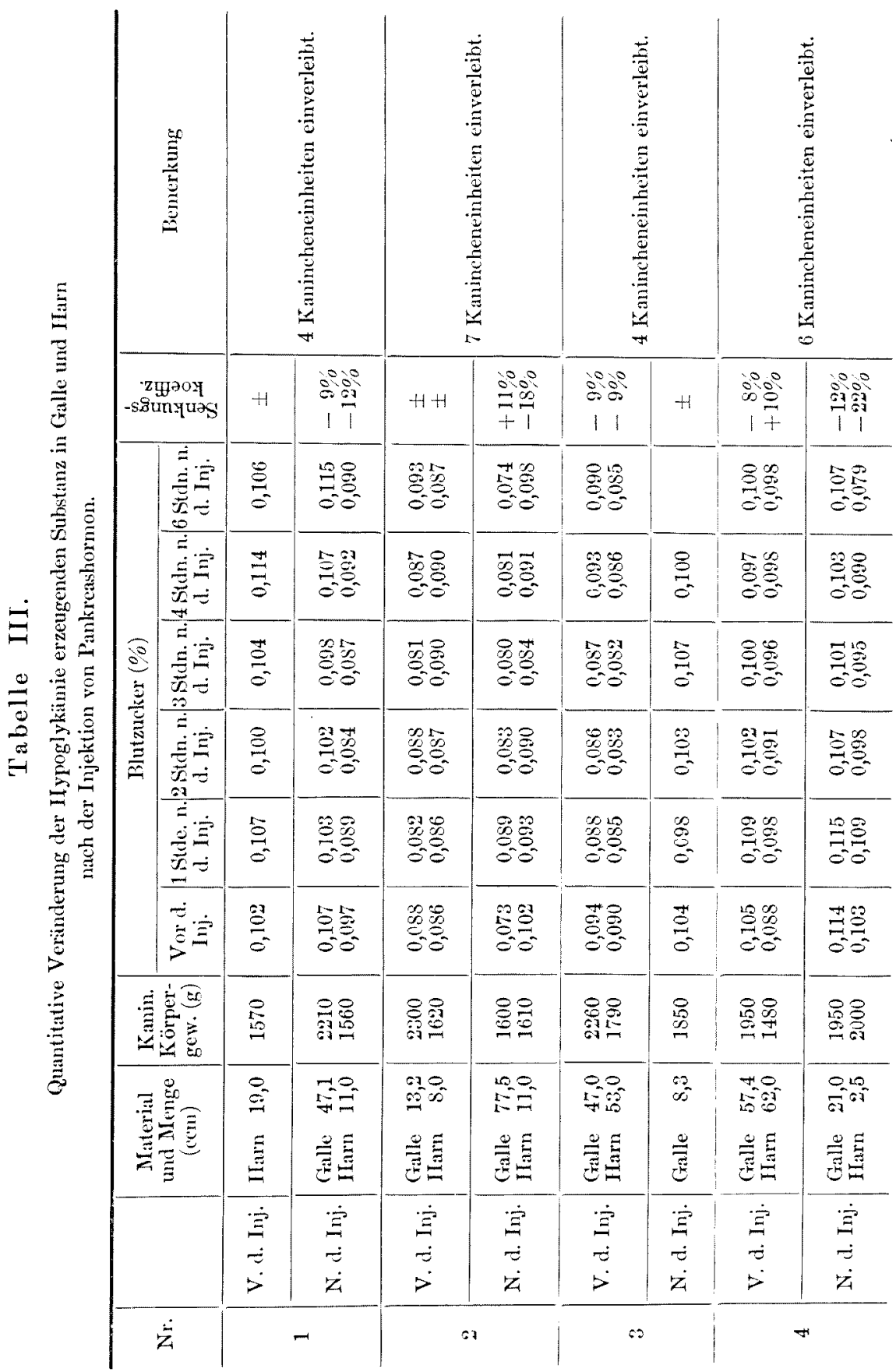


zur Galle, nach der Hormoninjektion sich stark vermehrt. Zum Beispiel ist Fall 4 am auffallendsten, in welchem das Extrakt aus nur 2,5 ccm Harn nach der Injektion 22\%ige Hypoglykämie hervorrufen konnte.

Diese Ergebnisse sprechen dafür, dass das einverleibte Pankreashormon beim Überschuss nicht in die Galle, sondern vorwiegend in den Harn ausgeschieden wird.

\section{Reduzierende Substanzder Galle.}

Seit langer Zeit ist es ein Problem der Physiologen, festzustellen, ob der Traubenzucker ein Bestandteil der normalen Galle sei. Mosler, ${ }^{\text {, }}$ Charcot, $\left.{ }^{5}\right) \mathrm{Kreh}^{(5)}$ und Brauer ${ }^{7)}$ behaupteten, dass die Galle im normalen Zustand keinen Traubenzucker enthält. Harley ${ }^{8)}$ hatte dagegen mitgeteilt, dass der Traubenzucker in der Galle von Ochs, Hund und Menschen immer nachweisbar ist. $\mathrm{Nauny} n^{9}$ war der Ansicht, dass die Glukose in der Blasengalle bald fehle, bald vorhanden sei, während sie in der von Ductus choledochus ansfliessenden frischen Galle immer enthalten sei, doch sei der Gehalt an derselben so klein, dass er ohne besondere Behandlung nicht nachgewiesen wird und dass der Traubenzucker nach dem Zuckerstich in der Galle immer vorkomme, was aber Brauer auf Grund seiner Experimente verneinte. Nach Hiray a ma ${ }^{10)}$ beträgt die Zuckermenge der Galle von Kaninchen, welchem Pilokarpin eingespritzt ist, zwischen $0,012 \%$ und $0,103 \%$, durchschnittlich $0,058 \%$. Nach $\mathrm{Nagai}{ }^{113}$ schwankt der Zuckerwert normaler Kaninchengalle zwischen 0,035\% bis $0,011 \%$. Wenn man solche Mitteilung durchsieht, fällt es ins Auge, dass der Zucker von Galle nur in sehr kleiner Menge enthalten ist und also nach der Empfindlichkeit der Methode und Genauigkeit der Ausführung der Bestimmung oft entgehen wird, folglich konnte man nicht zu einem übereinstimmenden Schluss kommen. All diese Arbeiten sind mit Reduktionsmethoden ausgeführt worden. So weit ich aus der mir zugänglichen Literatur ersehe, ist keine Untersuchung vorhanden, die den Beweis geliefert hat, dass die reduzierende Substanz der Galle wirklich Glukose ist. Ich unter-

\footnotetext{
4) Mosler, Zit. nach Brauer (7).

5) Charcot, Zit. nach Brauer (7).

6) Krehl, Zit. nach Brauer (7).

7) Brauer, Zeitschr. fur physiol. Chem., 1908, 40, 182.

8) Harley, Zit. nach Brauer(i).

9) Nauny n, Arch. f. exper. Path. u. Pharmak., 1875, 3, 85 u. 157.

10) H ir a y a ma, Tohoku Jour. of Exper. Med., 1924, 4, 507.

11) Naga i, Nihon Naikagakkai Zasshi, 1924, 12, 520 u. 632.
} 
nahm erstens zu erkennen, wie viel reduzierende Substanz in der Galle von Schwein und Kaninchen enthalten ist und zweitens nachzuweisen, dass dieselbe aus Zuckerarten besteht.

Beim Schwein wird die Galle aus frischen Gallenblasen ins Becherglas übertragen und durch Umrühren mit Glasstab vollst:̈ndig gemischt, weil sie auch in einer Blase verschiedene Konzentration besitzt, und ein Teil davon zur Bestimmung der reduzierenden Substanzen benutzt ist. Beim Kaninchen ist die Galle in oben genaunter Weise gewonnen. Die Bestimmung ist nach der neuen Ba ng'schen Methode ${ }^{12 ?}$ ausgeführt. Hierbei muss man darauf acht geben, dass bei Antrendung von zu viel Galle eine Trübung der Extraktionsflüssigkeit erscheint, was bei der genauen Bestimmung störend rirkt.

\section{Tabelle IV.}

Reduzierende Substanz der Galle $(0, g)$.

\begin{tabular}{|c|c|c|c|}
\hline Tier & \multicolumn{2}{|c|}{ Schwein } & Kaninchen \\
\hline $\begin{array}{l}\text { Gebalt an der } \\
\text { reduzierenden } \\
\text { Substanz }\end{array}$ & $\begin{array}{l}0,0483 \\
0,0729 \\
0,0227 \\
0,0315 \\
0,0287 \\
0,0453 \\
0,0302\end{array}$ & $\begin{array}{l}0,0243 \\
0,0875 \\
0,0275 \\
0,0819 \\
0,0272 \\
0,0207\end{array}$ & $\begin{array}{l}0,0377 \\
0,0192 \\
0,0185 \\
0,0166 \\
0,0245\end{array}$ \\
\hline Durchschnitt & \multicolumn{2}{|c|}{0,0806} & 0,0233 \\
\hline
\end{tabular}

Aus der Tabelle IV sieht man, dass die reduzierende Substanz der Galle beim Schwein zwischen 0,0207 \%o und 0,0722\% und beim Kaninchen von $0,0166 \%$ bis $0,0377 \%$ schwankt, d. h. durchschnittlich $0,0306 \%$ beim ersteren und $0,0233^{\circ}, o$ beim letzteren zeigt. Wenn man die reduzierende Substanz von zweierlei Tieren vergleicht, so liegt die von Schwein etwas höher als die von Kaninchen. Dass aber die reduzierende Substanz nach Bang'scher Methode durch Injektion von Pankreashormon parallel mit Blutzucker allmählich vermindert, wie spïter mitgeteilt wird, deutet gerade darauf hin, dass diese reduzierende Substanz höchstwahrscheinlich nichts anderes als Zucker ist. Ferner konnte ich bestätigen, dass dice reduktionsfähige Substanz tatsüchlich Zucker ist, indem ich auf Glukosizonbildung, Reduktions-, Polarisationsvermögen und Vergährung prüfte.

12) Bang, Mikromethoden z. Blutuntersuchung. Miinchen u. Wiesbaden, 192255. 
500-900 ccm Galle wurde aus mehreren Gallenblasen gesammelt und nach Patein und Dufau'scher Methode, welche von Kumagai und

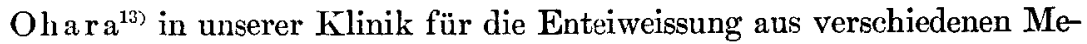
dien mit kleiner Modifikation angewandt wurde, enteiweisst. Um die Salze, welche eigentlich in der Galle enthalten sind und bei der Enteiweissungsprozedur zugebracht werden, möglichst zu entfernen, hatten wir das Filtrat nach Enteiweissung durch Vakuumdestillation vollständig abgedampft. Leicht erwärmter Methylalkohol wird in den Kolben eingegossen, stark umgerührt, und dann abfiltriert. Man verjagt Alkohol, indem man das Fjltrat in Vakuum abdampft, und danach mit destilliertem Wasser auf 1/51/10 Volum der originellen Gallenmenge verdünnt. Diese kondensierte Flüssigkeit benutzten wir in folgender Weise.

1. Glukosazonbildung. Man nimmt in jedem Fall $20 \mathrm{~cm}$ von der zu prüfenden Lösung und bringt dazu $0,5 \mathrm{~g}$ salzsaures Phenylhydrazin und I $\mathrm{g}$ essigsaures Natrium hinein, filtriert und erhitzt das Filtrat auf dem Wasserbad ca. 1 Stunde lang. Nach der Abkühlung kann man schön nadelförmiges Glulsosazonkristall bei Anwesenheit von Zucker mikroskopisch nachweisen.

2. Das Reduktionsvermögen wird nach Momos $\mathrm{e}^{14)}$ bestimmt.

3. Zur Beobachtung der Polarisation diente uns ein Polarimeter von Schmidt$\mathrm{H}$ ä $\mathrm{sch}$, der bis auf $0,01^{\circ}$ genau abzulesen gestattet.

4. Die Vergührung wird mit Bierhefe ausgeführt. Jedenfalls ist Zuckerlösung und destilliertes Wasser zur Kontrolle angewandt.

Zuerst hatten wir die Empfindlichkeit der Phenylhydrazinreaktion gegen Traubenzucker geprüft. Wir stellten $0,2 \%$ ige Traubenzuckerlösung als Stammlösung dar, sie zehnfach und dann doppelt verdünnend, so dass 0,02-, 0,01-, 0,005-, 0,0025- und 0,00125\% ige Zuckerlösungen bereitet wurden. Das schön typische Glukosazon wird aus der Lösung von 0,02-, $0,01-$ bis $0,005 \%$ iger Konzentration auffallend, aus der von $0,0025 \%$ iger ganz spärlich und aus der von 0,00125\% iger keinesfalls gebildet. Da 20 cem Lösung zur Reaktion gebraucht wird, kann man $0,0025 \times \frac{20}{100}=$ 0,0005 g Traubenzucker nach dieser Reaktion nachweisen.

Diese chemische Reaktion kann durch zugleich enthaltene verschiedene andere Substanzen abgeschwächt oder ganz gestört werden. Darum hatten wir zu erforschen versucht, ob diese Enteiweissungsmethode irgendeinen Einfluss auf die Phenylhydrazinreaktion, Reduktions-, Polarisationsund Gährungsvermögen ausübt. Wie schon angezeigt ist, beträgt die reduzierende Substanz der Galle nach Bang'scher Methode durchschnitt-

13) Kumagai u. Obara, Chugai Iji Shinpo, 1925, 1073, 1.

14) M omose, Tôkyô Igakkai Zasshi, 1915, 29, 903. 
lich $0,0233 \%$ bei Kaninchen, und 0,0306\% bei Schwein. Deshalb stellten wir $0,02 \%$ ige Tranbenzuckerlösung her, die der ganz gleichen Prozedur wie bei Enteiweissung unterworfen und durch die Vakuumdestillation bis auf 1/10 Volum, d. h. 10 facher Konzentration der Originallösung eingedampft wird ; so muss diese letzte Lösung demnach Traubenzucker zu 0,2\% enthalten, womit ich auf Phenylhydrazinrealition, Redultions-, Polarisations- und Gährungsfïhigkeit prüfte. Was die Osazonbildung anbetrifft, so sieht man dieselbe bei 0,005\% iger Lösung, was etwas schwächere Reaktionsfähigkeit als reine Traubenzuckerlösung zeigt. Der Zuckergehalt dieser konzentrierten Lösung nach Reduktions- und Polarisationsvermögen betrïgt $0,163 \%$ resp. $0,152 \%$, umgerechnet für Originallösung je $0,0163 \%$ und $0,0152 \%, \mathrm{~d} . \mathrm{h}$. es ist $0,0037 \%$ iger Verlust nach dem ersteren und $0,0048 \%$ iger nach dem letzteren zustande gekommen. Die Gührungsprobe mit Bierhefe fiel stark positiv aus.

Ferner stellten wir zur Kontrolle einen Versuch mit Galle, die Traubenzucker zu $0,1 \%$ enthält, an. Das Resultat ist im folgenden kurz angezeigt.

\begin{tabular}{l|c|c|c|c|c}
\hline & $\begin{array}{r}\text { Osazon- } \\
\text { bilkung } \\
\text { bis }\end{array}$ & $\begin{array}{c}\text { Reduzier } \\
\text { Kraft (um- } \\
\text { gerechnet } \\
\text { für Original- } \\
\text { lösung) }\end{array}$ & $\begin{array}{c}\text { Polarisation } \\
\text { (umgerechnet } \\
\text { fir Original- } \\
\text { lösung) }\end{array}$ & Gährung \\
\hline 1. & $\begin{array}{c}1,72 \text { fach rerdiunntes Filtrat } \\
\text { nach der Enteiweissung }\end{array}$ & $\begin{array}{c}0,0025 \% \\
(+)\end{array}$ & $0,1256 \%$ & $0,1309 \%$ & nicht \\
versucht
\end{tabular}

Aus der Tabelle erkennt man folgendes: Die Osazonbildung bekommt nach der Kondensation leichtgradige Störung, und Reduktionsvermögen zeigt bei 1 und 2 mässig und bei 3 nur gering höheren Wert als $0,1 \%$, was die Verminderung der reduzierenden Substanz wïhrend der Kondensation bedeutet. Woher kommt dieser höhere Wert? Sicherlich muss dieser Unterschied auf den in der Galle enthaltenen reduzierenden und drehenden Substanzen beruhen, und dieser Überschuss entspricht gerade der Reduktion nach Bang'scher Methode. Bezüglich der Gährungsprobe kann man keine Hemmung beobachten. 
Resultat bei der Schweinegalle.

$500 \mathrm{ccm}$ Galle aus 7 Stücken. Nach der Enteiweissung gewannen wir $840 \mathrm{ccm}$ Lösung, die 2 fach verdünnt wurde. Die Flüssigkeit wurde bis $\frac{1}{5}$ Volum der originellen Gallenmenge kondensiert.

1. Polarisation : $+0,04^{\circ}$, als Glukose $0,0762 \%$, umgerechnet für originelle Galle $0,0152 \%$;

2. Reduktion : als Glukose $0,096 \%$, umgerechnet für originelle Galle 0,0192\%;

3. Glukosazonbildung mit $20 \mathrm{ccm}$ : $(+)$;

4. Vergährung: $(+)$.

Aus den obigen Ergebnissen ist einwandlos hervorgegangen, dass der Traubenzucker ein Bestandteil der normalen Galle ist.

Die Gallensekretion.

Dobreff ${ }^{15)}$ hat beschrieben, dass sich dic Gallensekretion bei im Hunger befindlichen Hund durch Pankreashormon steigert und zwar nach 30-60 Minuten das Maximum erreicht, um danach in anderthalb oder 2 Stunden bis zum Anfangswert zurückzukehren. Brugsch und Horster ${ }^{16)}$ berichteten auch analoges Verfahren ; sie konnten nümlich am Hunde mit 20 Einheiten Pankreashormon 87\% ige Sekretionszunahme herbeiführen. Ausserdem kommt Sakurai ${ }^{17)}$ zum gleichen eindeutigen Schluss der genannten Autoren. Wir studierten den Einfluss des Pankreashormons auf die Sekretion von Galle an Kaninchen, welches für unsere Arbeiten am zweckmässigsten ist, da die Galle bei Grasfressern immer fortdauernd in den Darm fliesst.

Gallensekretion im normalen Zustand.

Zuallererst muss man die Gallensekretion im normalen Zustand kennen. In der oben genannten Weise wird die Gallenausscheidung beim auf dem Operationstisch gefesselten Kaninchen während langer Zeit beobachtet.

Fall 1. Kaninchen, $2150 \mathrm{~g}$. Operationsdauer 20 Minuten. 4 Stunden hindurch wird die Sekretionsveränderung studiert. Schon nach 2 Stunden sieht wan auffallende Verninderung der Selretion, sie nimmt nümlich als Stundenmenge von $8,2 \mathrm{ccm}$ bis $5,8 \mathrm{ccm}$ ab.

15) Dobreff, Bioch. Zeitschr., 1924, 154, 364.

16) Brugsch und Horsters, Med. Klinik, 1924, 661.

17) Saku rai, Tôkyô Igakkai Zasshi, 1926, 40, 928. 
Fall 2. Kaninchen, $2000 \mathrm{~g}$. Operationsdaver 14 Minuten. S Stunden lang erfolgt die Gallensekretion. Die Gallenmenge ist in Laufe der Zeit allmühlich rermindert wie beim vorigen. Der Blutzucker zeigt nach 8 Stunden 2 fach grösseren Wert als den vor der Operation.

Wie die folgende Tabelle zeigt, nimmt die Sekretion mit dem Verlauf der Zeit ab, was wahrscheinlich von allgemeiner Schwäche durch Inanition und Operation, d. h. von herabgesetzter I,ebensfähigkeit verursacht wird.

$$
\text { Tabelle V. }
$$

Gallensekretion bei Kaninchen in normalem Zustand.

\begin{tabular}{|c|c|c|c|c|}
\hline & $\mathrm{Nr}$. & Zeit & Gallenmenge $(\mathrm{ccm})$ & Blutzucker $(\%)$ \\
\hline 1 & $\begin{array}{l}\text { Q, } 2150 \mathrm{~g} . \\
\text { Operation } \\
20 \text { Minuten }\end{array}$ & $\begin{array}{l}5^{\mathrm{h}} 2^{\prime}-6^{\mathrm{h}} 2^{\prime} \\
6^{\mathrm{h}} 2^{\prime}-7^{\mathrm{b}} 2^{\prime} \\
7^{\mathrm{h}} 2^{\prime}-8^{\mathrm{h}} 2^{\prime} \\
5^{\mathrm{b}} 2^{\prime}-9^{\mathrm{b}} 2^{\prime} \\
9^{\mathrm{h}} 2^{\prime}-9^{\mathrm{h}} 32^{\prime}\end{array}$ & $\begin{array}{l}8,2 \\
5,8 \\
5,3 \\
5,6 \\
2,3\end{array}$ & \\
\hline 2 & $\begin{array}{c}\text { P, } 2000 \\
\text { Operation } \\
14 \text { Minuten }\end{array}$ & 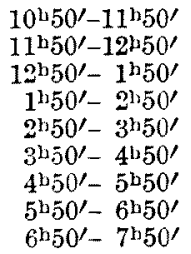 & $\begin{array}{r}10,0 \\
9,9 \\
10,8 \\
10,2 \\
9,9 \\
9,2 \\
8,5 \\
6,7 \\
4,9\end{array}$ & $\begin{array}{l}0,086\left(10^{\mathrm{h}} 30^{\prime}\right) \\
0,192\left(1^{\mathrm{h}} 55^{\prime}\right) \\
0,172\left(5^{\mathrm{h}} 0^{\prime}\right) \\
0,180\left(7^{\mathrm{h}} 30^{\prime}\right)\end{array}$ \\
\hline
\end{tabular}

Gallensekretion bei Pankreashormoninjektion.

Es ist bekannt, dass der Blutzuckergehalt beim Kaninchen durch Fesselung oder Operation eine merkliche Erhöhung erführt. Bei unseren Experimenten, bei welchen Kaninchen während mehrerer Stunden auf dem Operationstisch festgebunden werden und die Bauchhöhle aufgemacht werden muss, zeigt auch der Zuckergehalt des Blutes 8 Stunden nach der Operation noch 2 fach grösseren Wert als den Anfangswert, wie schon angegeben ist. Also bedarf es in diesem Falle einer viel grösseren Menge Pankreashormon als im normalen Zustand, um die Wirkung desselben genügend entfalten zu lassen. Wir spritzten Pankreashormon bei mehrmaliger Einteilung subkutan ein.

Fall 1. Kaninchen, $2900 \mathrm{~g}$. Operationsdauer 20 Minuten. Pankreashormon $4 \mathrm{Ka}$ nincheneinheiten. Vor der Injektion ron Pankreashormon betrïgt die Gallenmenge durchschnittlich 2,4 ccm pro 20 Minuten und die nach der Einverleibung ron Pankreashormon vermehrt sich allmählich 60-80 Minuten danach bis zum Maximum, 3,4 ccm, d. h. 30\%ige Zunahme der Sekretion. Zum zweiten Mal, 3 Stunden nach der ersten Injektion, wird 1 Kanincheneinheit und abermals $1 \mathrm{Kanincheneinheit} 1$ Stunde danach subkutan appliziert, 
wodurch man jedoch keine Vermehrung der Gallenausscheidung konstatieren konnte. 4 Stunden nach der ersten Injektion kommt die Ausscheidungsmenge zum Ausgangswert zurück und vermindert sich danach mehr und mehr, was wahrscheinlich darauf zu beruhen scheint, dass durch Hunger und Operation die Lebenskraft allmählich abgeschwächt wird. Der Blutzuckerwert steigt wegen der Operation bis zu zweifach grösserer Menge, der aber nach der Injektion von Pankreashormon allmühlich sich vermindert. Der durch Operation erhöhte Zuckergehalt von Galle weist durch Pankreashormon ca. 50\% ige Herabsetzung auf.

Fall 2. Kaninchen, $2400 \mathrm{~g}$. Operationsdauer 9 Minuten. Pankreashormon $7 \mathrm{Ka}$ nincheneinheiten. Bei diesem Fall sieht man reichliche Absonderung der Galle in der ersten 20 Minuten, was wohl rom plötzlichen Ausfliessen der Flüssigkeit, welche sich durch Unterbindung von Ductus choledochus wïhrend der Operation staut, herkommen muss. Die Gallenmenge betrïgt durchschnittlich $3,3 \mathrm{ccm}$ auf 20 Minuten vor der Hormoninjektion. Von 60 bis 80 Minuten nach der ersten subkutanen Injektion von 2 Kanincheneinheiten Pankreashormon wird 4,9 ccm und nach der zweiten $5,2 \mathrm{ccm}$ Galle pro 20 Minuten sezerniert, d. h. 55\%ige Zunahme. Danach aber neigt sie zur Verminderung trotz der Hormoninjektion. Der Blutzucker erhöht sich zweifach durch die Operation, aber ernjedrigt nach der Hormonapplikation bis 0,035\%. Die Zuckermenge der Galle erführt keine Vermehrung durch die Operation, doch 50\%ige Senkung durch die Hormoninjektion.

Beim Durchschen dieser Versuche fällt uns auf, dass das Pankreashormon auf die Gallensekretion steigernd wirkt und diese Zunahme nach ca. 80 Minuten das Maximum, nümlich 40-50\%, erreicht, um danach allmählich mit dem Laufe der Zeit abzunehmen, was die Abschwächung der Lebenskraft des Tiers durch die Operation und Inanition bedingt. Das Pankreashormon wird die Funktion der Leber reizen und folglich die Gallensekretion zur Vermehrung bringen. Die Verminderung der reduzicrenden Substanzen nach Bang'scher Methode nach der Pankreashormonapplikation spricht dafür, dass dieselbe überhaupt aus Zucker besteht.

Aus obengenannten Versuchen erkemnt man schon die Steigerung der Gallenabsouderung durch das Pankreashormon. Beruht dies aber nicht auf dem als Lösungsmittel des Pankreashormons eingeführten Wasser, wie Zunahme der Harnmenge bei Wasserzufuhr? Zur Kontrolle hatte ich dem Kaninchen gleiche Menge von physiologischer Kochsalzlösung unter ganz gleichen Bedingungen eingespritzt. Das Resultat ist in der Tabelle IV zusammengestellt, woraus man ersieht, dass dadurch keine Vermelhrung der Gallensekretion zustande kommt, und dass sowohl Blutzucker als auch Gallenzucker während mehrerer Stunden crhöht bleibt.

\section{Zusammenfassung.}

1. Das Pankreashormon wird nicht in die Galle, sondern in den Harn ausgeschieden. 


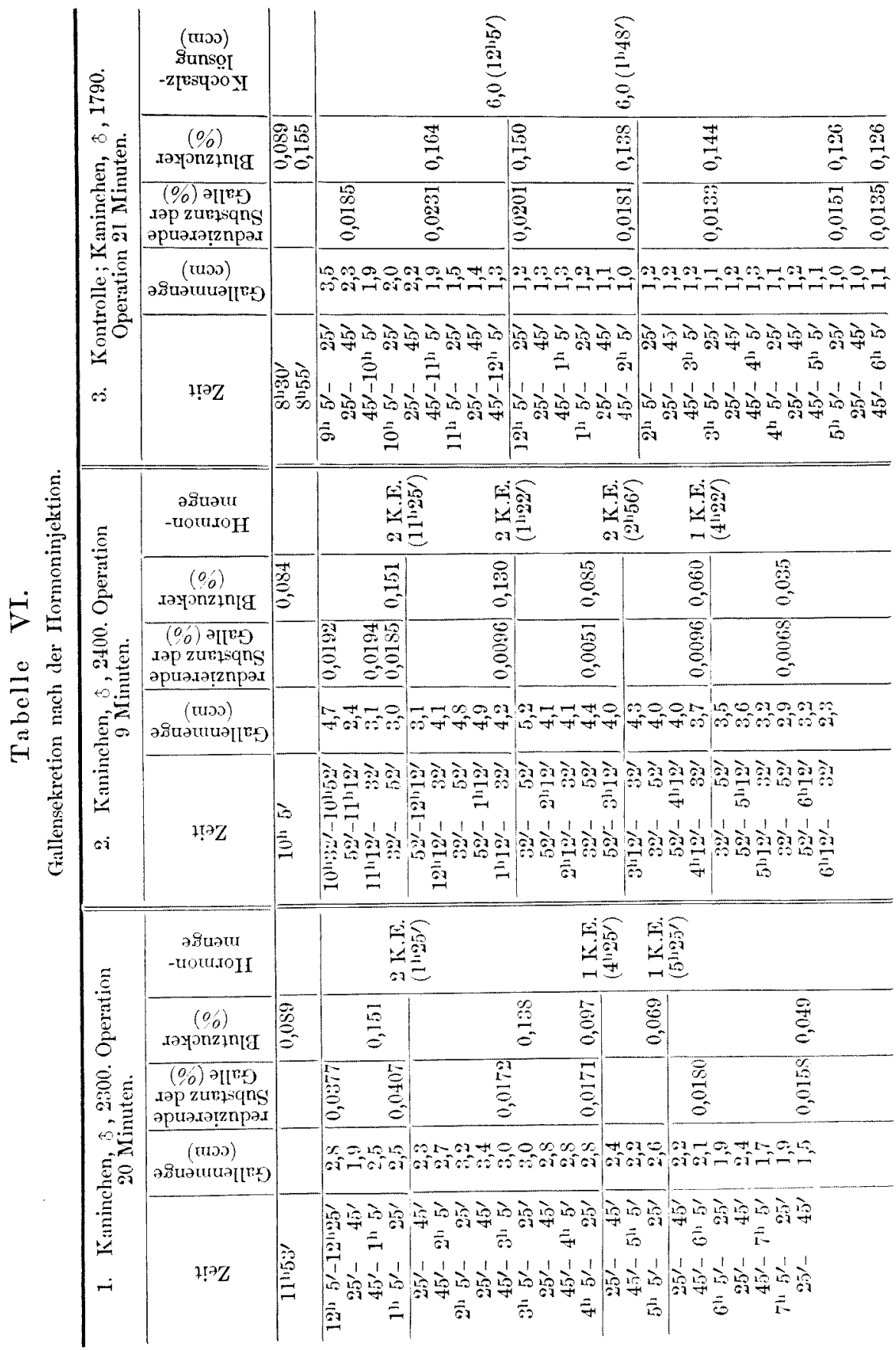


2. Die blutzuckersenkende Substanz ist auch in der Galle vom normalen Kaninchen oder Schwein nachweisbar.

3. Die reduzierende Substanz der Galle besteht hauptsächlich aus Glukose und wird durch Injektion des Pankreashormons vermindert ; mit anderen Worten ist der Traubenzucker ein Bestandteil von normaler Galle. 4. Das Pankreashormon beschleunigt die Gallensekretion.

Die Kosten für diese Untersuchung sind von der Sa i to-Hôonkai-Stiftung bestritten worden, wofür ich hierbei den besten Dank ausspreche.

Prof. T. Kumagai. 